Review

\title{
Role of Nutrition in the Pathogenesis and Prevention of Non-alcoholic Fatty Liver Disease: Recent Updates
}

\author{
Rahim Ullah ${ }^{1,2}$, Naveed Rauf1,2, Ghulam Nabi³, Hamid Ullah4, Yi Shen², Yu-Dong Zhou ${ }^{2}{ }^{\bowtie}$, Junfen Fu' ${ }^{1 凶}$ \\ 1. Department of Endocrinology, Children's Hospital, Zhejiang University School of Medicine, Hangzhou 310051, China \\ 2. Department of Neurobiology, Institute of Neuroscience, and the Collaborative Innovation Center for Brain Science, Key Laboratory of Medical \\ Neurobiology of the Ministry of Health of China, Zhejiang University School of Medicine, Hangzhou 310058, China \\ 3. Institute of Hydrobiology, the Chinese Academy of Sciences, Wuhan, 430072, China \\ 4. Laboratory of Reproductive Neuroendocrinology, Department of Animal Sciences, Faculty of Biological Sciences, Quaid-i-Azam University, Islamabad, \\ Pakistan \\ $\bowtie$ Corresponding authors: Prof. Zhou Yu-Dong; email: yudongzhou@zju.edu.cn, Phone number: 0086-057188208547, Prof. Junfen Fu; email: fjf68@qq.com, or \\ fjf68@zju.edu.cn, Phone number: 0086-571-88873013 \\ (c) Ivyspring International Publisher. This is an open access article distributed under the terms of the Creative Commons Attribution (CC BY-NC) license \\ (https://creativecommons.org/licenses/by-nc/4.0/). See http://ivyspring.com/terms for full terms and conditions.
}

Received: 2018.09.21; Accepted: 2018.11.24; Published: 2019.01.01

\begin{abstract}
Non-alcoholic fatty liver disease (NAFLD) is an acquired metabolic disease characterized by triglycerides (TGs) deposition in liver induced by other factors rather than alcohol consumption. NAFLD significantly contributes to liver diseases in children and adults. NAFLD pathogenesis is associated with age, gender, race and ethnicity. Insulin resistance, hyperinsulinemia, elevated plasma free fatty acids (FFAs), fatty liver, hepatocyte injury, liver inflammation, oxidative stress, mitochondrial dysfunction, imbalanced pro-inflammatory cytokines, and fibrosis are the characteristics of NAFLD. Factors including genetic and epigenetic pathways, sedentary lifestyle, sleep, and diet composition affect NAFLD pathogenesis. In this review, we discuss the aetiology, risk factors and pathogenesis of NAFLD. Special focus is given to macro and micro nutrition as causing factors and their role in the prevention of NAFLD pathogenesis.
\end{abstract}

Key words: nutrition, hepatic inflammation, NAFLD pathogenesis, prevention

\section{Introduction}

NAFLD is an acquired metabolic disease induced by metabolic stress and characterized by fat deposition in liver. NAFLD is associated with insulin resistance and genetic susceptibility and has overlapping histological characteristics with alcoholic liver [1]. NAFLD has four stages including hepatic fat deposition (hepatic steatosis), hepatic fat deposition with inflammation, fibrosis and cirrhosis (Fig. 1). The first stage is hepatic fat deposition also called non-alcoholic fatty liver (NAFL). The second stage is non-alcoholic steatohepatitis (NASH), characterized by excess hepatic fat deposition and inflammation. Persistent liver inflammation (hepatitis) causes scar tissue formation in the liver and this stage is called fibrosis. The fourth stage is cirrhosis, which is a severe form of NAFLD where fibrosis replaces most of the liver cells and therefore the structure and function of liver cells are compromised. Cirrhosis then leads to liver failure. Hepatocytes work well in fibrosis, but are compromised in cirrhosis. The prevalence of adult NAFLD in the general population is $5 \%-20 \%$, whereas it is above $40 \%$ in obese and type 2 diabetic patients $[2,3]$. The prevalence of NAFLD in children is $3 \%$ but it increases up to $50 \%-70 \%$ among obese children [4]. NAFLD prevalence is $16.9 \%-23.8 \%$ in boys and $16.2 \%-22.6 \%$ in girls in developed countries whereas it is $8.1 \%-12.9 \%$ in boys and $8.4 \%-13.4 \%$ in girls in developing countries [5]. These data suggest that NAFLD is one of the most important metabolic diseases arising parallel with other metabolic disorders including obesity, diabetes and cardiovascular disease.

Nutrition is the principal contributory factor effecting NAFLD pathogenesis. It has been reported that different diet components affect the progression and development of NAFLD [6-15]. Therefore, it is 
very important to comprehensively discuss the role of nutrition in the progression, development and prevention of NAFLD. In this review, we discuss the risk factors and pathogenesis of NAFLD with a special focus on the role of nutrition in NAFLD pathogenesis and prevention.

\section{Risk factors and pathophysiology of NAFLD}

The development and progression of NAFLD were initially described by a "two hit" theory. The first hit, defined by "hepatic steatosis with or without hepatitis" is characterized by the presence of metabolic syndrome and TGs deposition in the liver. During the first hit, insulin resistance and hyperinsulinemia appear that alter hepatic pathways involved in the uptake, degradation, synthesis and secretion of free fatty acids (FFAs) that result in the deposition of fatty acids in the liver [16-18]. FFAs, Toll-like pattern recognition receptors (TLRs) and cytokines stimulate molecular pathways that block insulin signalling and thus induce insulin resistance. Insulin resistance is the result of overnutritioninduced inflammation that further supports NAFLD pathogenesis. The conditions of the first hit support



Figure 1. Convergence of fats and carbohydrates induced key signalling molecules on NAFLD pathogenesis. Here, fats and carbohydrates mean a high intake or intake of harmful components of fats and carbohydrates supporting NAFLD pathogenesis. Fats and carbohydrates directly or indirectly induce metabolic stress and related signalling cascade that collectively induce insulin resistance and support overall NAFLD pathogenesis. FFAs $=$ free fatty acids, IKK $\beta=$ inhibitor of $K B$ kinase $-\beta, N F-K B=$ nuclear factor kappa $B, M a c=$ macrophages, TNF- $\alpha=$ tumor necrosis factor- $\alpha, \mathrm{IL}-6=$ interleukin $6, \mathrm{IL}-1 \quad \beta=$ interleukin $1 \beta$, ER= endoplasmic reticulum, $\mathrm{IR}=$ insulin resistance, $\mathrm{TGs}=$ triglycerides, $\mathrm{NASH}=$ non-alcoholic steatohepatitis, $\mathrm{HSC}=$ hepatic stellate cells, KC= Kupffer cells. hepatocyte injury and the pathogenic process of the second hit. Liver inflammation, oxidative stress, mitochondrial dysfunction, imbalanced pro-inflammatory cytokines, and fibrosis are the characteristics of the second hit that lead to the development of NASH and fibrogenesis (Fig. 1) [17-20]. Currently, "multiple hit" theory explains the progression of NAFLD where multiple hits act together to support liver inflammation. Many factors including diet, lifestyle, parental diet, genetic, epigenetic, environmental factors, insulin resistance and type 2 diabetes, etc. collectively contribute to obesity and widespread metabolic complications appear during NAFLD pathogenesis [16, 21, 22] (Fig. 2).

Importantly, the complex interplay of hepatic resident cells and activated immune cells, such as Kupffer cells, $\mathrm{T}$ cells, and hepatic stellate cells, with additional inflammatory factors and (TLRs) [23-26] contributes to the development and progression of NAFLD (Fig. 1). Additionally, the chronic activation of hepatic stellate cells and apoptosis are considered the principal mediators of hepatic fibrosis and cirrhosis. Similarly, hepatic progenitor cells (HPCs) associated with NASH and fibrosis, are the resident hepatic stem cells showing expansion during pediatric NAFLD and are involved in hepatic response to oxidative stress [27]. Furthermore, evidence suggests that Kupffer cells mediate different actions in NAFLD pathogenesis including immune tolerance and lipid homoestasis [24, 28]. In this regard, Stienstra et al. reported that Kupffer cells trigger TGs deposition and hepatic steatosis through interleukin-1 beta (IL-1 $\beta$ )-mediated suppression of perioxisome proliferator-activated receptor-a (PPARa) actions [29]. Similarly, De Vito et al. (2012) reported that deletion of Kupffer cells prevents hepatic steatosis and liver damage [24]. Likewise, altered functionality of peripheral $\mathrm{T}$ cell subpopulations has been reported in NASH [23]. Compared to CD4+ and $\mathrm{CD} 20+$, a high proportion of CD8+ $T$ cell subpopulations have been reported in pediatric NAFLD. Additionally, these predominant CD8+ T cells in pediatric NASH were associated with up-regulated hepatic interferon- alpha, a high number of infiltrating neutrophils in association with reactive oxygen species (ROS) production in systemic neutrophils, 
and altered phenotype and functionality of circulating lymphocytes and neutrophils. Similarly, in adult $\mathrm{NASH}, \mathrm{CD} 8+$ cells were a minor component of natural killer cells $[23,30]$. Furthermore, up-regulated autonomic receptors on HSC have been reported in adult NAFLD patients [31, 32].

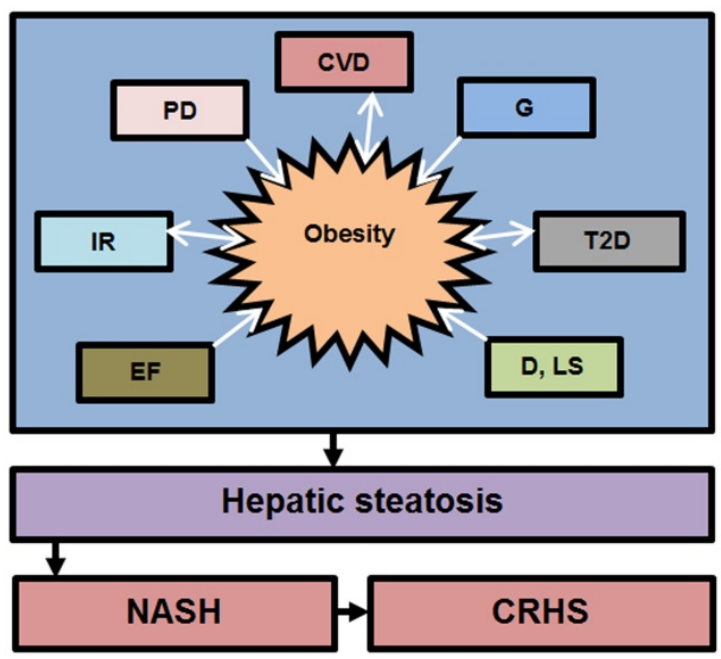

Figure 2. Contribution of various factors in the development of obesity and NAFLD. The above factors trigger NAFLD pathogenesis directly or indirectly through obesity. Abbreviation: $C V D=$ cardiovascular diseases, $G=$ genetics, $I R=$ Insuline resistance, $\mathrm{T} 2 \mathrm{D}=$ type 2 diabetes, $\mathrm{D}=$ diet, $\mathrm{LS}=$ lifestyle, $\mathrm{EF}=$ environmental factors, $\mathrm{PD}=$ parental diet, NASH= non-alcoholic steatoheptitis, $\mathrm{CRHS}=$ cirrhosis. Single sided arrows mean unidirectional, whereas double sided arrows mean bidirectional interaction.

On the basis of histology, NAFLD in children is similar to that in adults. However, the histologic distribution and progression of NASH in terms of inflammation and hepatocellular damage in children are different from those in adults [33, 34]. Liver biopsies from overweight and obese children (2-18 years of age) suffering from NAFLD revealed two different pathologic subtypes of NASH, subtype 1 (adult-type) and subtype 2 (pediatric-type) [35]. Histopathological features of NASH subtype 1 are steatosis, lobular inflammation, ballooning degeneration and presence or absence of perisinuoidal fibrosis, whereas the features of NASH subtype 2 are macrovesicular hepatocellular steatosis, portal inflammation, absence or minimal ballooning degeneration and presence or absence of portal fibrosis. Additionally, the prevalence of both subtypes differ with gender and race (for a review, see [36]). Although both subtypes overlap in $32 \%$ of patients, type2 NASH is the most predominant subtype [37]. Importantly, the etiopathogenesis, prognosis and differential response of both subtypes to treatments are still not clear and need further studies. NAFLD in children and adults has similarities and differences in the pathogenesis at the molecular and genetic level [38]. Similarly, the outcome also overlaps, however it is difficult to cover all of these in the current review; therefore interested readers can study the review article of Nobili et al. (2016) for detailed similarities and differences between NAFLD in children and adults [38]. The differences between NAFLD in adults and children are shown in Table 1.

Table 1. Differences between Children and Adult NAFLD

\begin{tabular}{|c|c|c|}
\hline Type of parameter & Adults & Children \\
\hline \multicolumn{3}{|l|}{ Histological features } \\
\hline Steatosis & $\begin{array}{l}\text { Typically mild to } \\
\text { moderate }\end{array}$ & Typically moderate to severe \\
\hline Ballooning & Common & Uncommon \\
\hline Inflammation & Mainly lobular & Mainly portal \\
\hline Fibrosis & Pericellular chicken wire & $\begin{array}{l}\text { Predominantly } \\
\text { portal-periportal }\end{array}$ \\
\hline \multicolumn{3}{|l|}{ Outcome } \\
\hline Cirrhosis & $5-10 \%$ & $1-2 \%$ \\
\hline $\mathrm{HCC}$ & Strong clinical evidence & Rare \\
\hline Metabolic syndrome & Strong clinical evidence & Strong clinical evidence \\
\hline Cardiovascular disease & Strong clinical evidence & Increased risk \\
\hline
\end{tabular}

\section{Contribution of macro and micro nutrients to the development of NAFLD}

The key problem of NAFLD and NASH is overnutrition and, therefore, after NAFLD diagnosis, a first focus should be given to controlling overfeeding and body weight. Weight loss ( $1 \mathrm{~kg} /$ week) is recommended for overweight (BMI $25-30 \mathrm{~kg} / \mathrm{m} 2$ ) and obese $(B M I>30 \mathrm{~kg} / \mathrm{m} 2)$ subjects and it seems unlikely that changes in the diet alone (without accompanying weight loss) might be sufficient to overcome NAFLD and NASH. However, it is important to note that rapid and uncontrolled weight loss is detrimental and even worsens NAFLD and NASH. We take two types of nutrients in our daily life: macronutrients and micronutrients.

\section{Macronutrients}

The nutrients that we take in large amounts in our daily life are macronutrients. Carbohydrates, fats and proteins are the principal types of macronutrients. Various chemicals exist in macronutrients that either support or prevent NAFLD pathogenesis. The role of macronutrients in NAFLD pathogenesis and prevention is summarized in Fig. 3.

\section{Carbohydrates}

Although the control of total caloric intake is very important to prevent metabolic complications including NAFLD induced by high energy intake, carbohydrates are also of great concern. NASH has been induced by a high carbohydrate diet in a desert gerbil [7]. Likewise, a carbohydrate rich diet is a major source of hepatic FFAs production in NAFLD subjects where it contributes to $30 \%$ FFAs production, whereas in normal subjects, high carbohydrate intake produces only 5\% FFAs [39]. Diets with a low carbohydrate 


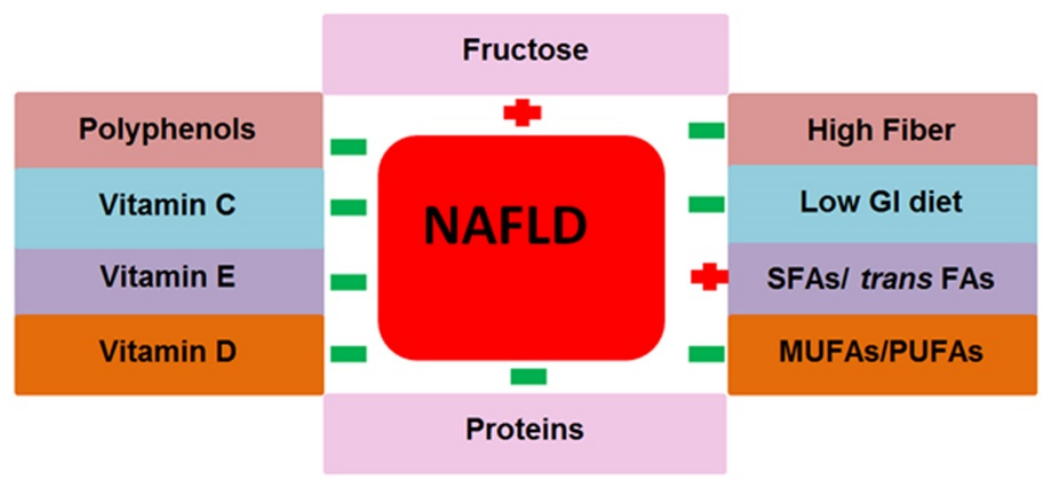

Figure 3. Contribution of macro and micronutrients in NAFLD pathogenesis. The above nutrition types support or prevent NAFLD pathogenesis and NAFLD related metabolic complications. Abbreviations: NAFLD= non-alcoholic fatty liver disease, $\mathrm{Gl}=$ glycemic index, SFAs= saturated fatty acids, trans FAs= trans fatty acids, MUFAs= monounsaturated fatty acids, PUFAs= polyunsaturated fatty acids. Positive $(+)$ means to support NAFLD pathogenesis, Negative $(-)$ means to prevent NAFLD. pop, Coca-Cola and tonic are soft drinks rich in fructose. A normo-caloric diet with a $3 \mathrm{~g}$ fructose $/ \mathrm{kg}$ of body weight per day increases hepatic fat deposition and serum TG levels and reduces insulin sensitivity in adult men [49]. Consumption of more than 1 soft drink (about 360ml)/day increases the risk for the development of metabolic syndrome, however this risk was not observed in subjects who were consuming less than 1 soft drink/day [45, 50].

\section{Fiber and glycemic index diet}

Fibers can be classified in two types: soluble and insoluble fibers. The examples of soluble fibers are pectin and content $(<45 \%$ of carbohydrates/day) have been reported to be helpful in improving weight loss and the overall metabolic profile and reduce intrahepatic TGs content [40]. Low carbohydrate intake improves the condition of NAFLD [41, 42]. Additionally, a low carbohydrate diet reduces TGs and increases HDL levels [43]. Paradoxically, consumption of a low carbohydrate diet over a long period stimulates NAFLD pathogenesis and glucose intolerance in an animal model [40] and increases total and low density lipoproteins (LDL) cholesterol in humans [43]. Interestingly, a low carbohydrate diet is helpful in weight loss if consumed for a short period (six months) but this weight loss cannot be maintained after one year. Importantly, the quick and rapid weight loss supports the disease progression in some NAFLD subjects [40]. Similarly the composition of ingested carbohydrates is also important. Here, we will discuss the role of some special carbohydrate types in the pathogenesis and prevention of NAFLD.

\section{Fructose}

As a sweetener, fructose has been used in juices, jellies and jams. Fructose can easily induce metabolic complications in children with NAFLD compared to children without NAFLD [44]. Fructose enriched soft drinks induce insulin resistance and support NAFLD development. Fructose intake causes lipogenesis, TGs synthesis and studies in ducks and rats have shown that it also causes hepatic steatosis [45, 46]. Additionally, high fructose intake also inhibits leptin (fat derived anorexigenic hormone) secretion, and therefore satiety cannot be achieved [47]. Similarly, a high fructose diet reduces PPARa activity and hepatic lipid oxidation and stimulates NF-kB expression that leads to oxidative stress, hepatic steatosis and hepatic fibrosis in rats [48]. Fructose interacts with transcriptional factors and affects the gene expression involved in glycolysis and lipogenesis [6]. Soda, soda gums. Pectin exists in fruits and gums exist in oat, beans, barley, lentils, peas, and chickpeas. Examples of insoluble fibers are cellulose (wheat), hemicellulose (grains) and lignin (vegetables). Insoluble fibers promote satiety (restrict caloric intake), whereas soluble fibers slow stomach emptying and glucose and cholesterol uptake. Soluble fibers from sources such as oat reduce TC levels [51, 52]. A recent meta-analysis study reviewed the effects of high fiber and low-glycemic index (GI) foods on glycemic response and cholesterol concentration [53] and suggested that such diet components might be beneficial for subjects who have an impaired insulin response. Low-GI foods or slow release carbohydrates induce a "second-meal effect" which is a reduced glucose response to the subsequent meal, and was described in early 1980 [54]. It was reported that a low-GI diet breakfast, a breakfast consisting of high-fiber indigestible and fermentable starch, induces the second-meal effect and reduces circulating FFAs and insulin levels after the subsequent meal in both men and women [55]. Of note, we cannot generalize the results of the above study to the long term effects of such diets on blood glucose, insulin and lipid profile but it does suggest that the inclusion of high-fiber indigestible and fermentable starch and low-GI carbohydrates might be helpful to maintain blood glucose, insulin and FFAs profile in subjects with insulin resistance and NASH.

From the above discussion it can be speculated that in the development of NAFLD and other hepatic complications, carbohydrate's composition is also important. However, more pre-clinical and clinical trials are suggested to investigate the role of different components of carbohydrates in the progression and treatment of NAFLD. 


\section{Fat}

A fat rich diet induces hepatic steatosis [9]. Fat accumulation triggers lipolysis in adipocytes and increases FFAs that result in reduced plasma adiponectin levels, attenuated lipid clearance from plasma and increased beta-oxidation in muscles [56]. In a previous study, we developed a rabbit model of pediatric NASH and found that HFD feeding increases body weight, liver weight and ALT, TG, IL-6, TNF-a levels, whereas it reduces serum adiponectin and IL-10 levels [57-60]. Similarly, in another study, we reported elevated levels of hyaluronic acid, serum ferritin, serum type III procollagen, ALT and aspartate aminotransferase (AST) in obese NAFLD and NASH children [61]. Fats are a diverse class of organic compounds; however, we will focus on some fat types involved in the pathogenesis and prevention of NAFLD.

\section{Saturated fatty acids (SFAs)}

SFAs are fatty acids that have only carbon to carbon single bonds (no double bond) in their hydrocarbon backbone. SFAs induce endoplasmic reticulum (ER) stress and hepatocyte injury in rats [62]. SFAs induce hypothalamic inflammation that leads to obesity onset and its related metabolic complications [63-65]. Dietary data revealed that NASH subjects took $14 \%$ of their total energy from SFAs, whereas it was $10 \%$ in control subjects. Intake of more than $10 \%$ of total energy from SFAs may support insulin resistance, whereas less than $10 \%$ of total energy from SFAs reduces plasma LDL and TG levels; however, less than $7 \%$ of total energy from SFAs does not further improve NASH but is even detrimental.

\section{Monounsaturated fatty acids (MUFAs)}

MUFAs are a class of fatty acids that have only one carbon to carbon double bond in their hydrocarbon backbone, with the rest of the bonds being single bonds. MUFAs exist in some foods including nuts, olive oil, and avocados. Diet driven MUFAs decrease oxidized LDL [66], LDL cholesterol [67], TC and TGs levels with no decrease in HDL [68-70]. Replacement of carbohydrates and saturated fats with MUFAs increases HDL and reduces glucose and blood pressure in diabetic subjects [71]. Additionally, another study reported that compared to a high-carbohydrate diet $(28 \%$ of total energy intake), a diet rich in MUFAs (40\% of total energy intake) extensively decreases VLDL triacylglycerol and VLDL cholesterol and is acceptable for T2D subjects [71]. Additionally, it has been reported that a diet composed of $20 \%$ MUFAs as an energy source of the total daily caloric intake increases fatty acid oxidation through activated PPARs activity and reduces lipogenesis through diminished sterol regulatory element binding protein (SREBP) activity in NAFLD men and women [72]. Regardless of physical exercise, a high MUFA intake significantly reduced hepatic fat content in T2DM subjects [72]. The above evidence collectively suggests that replacement of a SFA and carbohydrate rich diet with a MUFA rich diet might be beneficial in NAFLD treatment.

\section{Polyunsaturated fatty acids (PUFAs)}

PUFAs are a class of fatty acids that have two or more than two carbon to carbon double bonds in their hydrocarbon backbone. PUFAs are present in sea fish, green leafy vegetables, rapeseed oil and flax seeds, and are beneficial against NAFLD. Interestingly, 50 $\mathrm{g} /$ day intake of rapeseed/canola oil for four weeks has been shown to improve TC, LDL, and hepatic enzymes up to a healthier range in obese men compared to those who ingest $50 \mathrm{~g} /$ day of olive oil [73].

Omega-3 ( $\omega-3)$ and omega-6 ( $\omega-6)$ are important PUFAs and have a role in NAFLD pathogenesis. $\omega-3$ fatty acids are beneficial, however $\omega-6$ fatty acids should be avoided because of their ability to increase inflammatory markers [11]. It has been reported that patients with NASH have a high intake of $\omega-6$ fatty acids and an abnormal $\omega-3 / \omega-6$ fatty acids ratio [74]. Therefore the recommended $\omega-3$ to $\omega-6$ ratio should be $1: 1$ to $1: 4$. $\omega-3$ fatty acids regulate gene expression involved in hepatic lipid metabolism, fatty acid oxidation and reduced expression of pro-inflammatory genes. Docosahexaenoic acid (DHA), linolenic acid (plant oil), and eicosapentaenoic acid (EPA) [fish oil] are examples of $\omega-3$ fatty acids. It has been reported that $\omega-3$ fatty acids have beneficial effects against hepatic steatosis and inflammatory markers and improve insulin sensitivity and cardiovascular disease [75-77]. Similarly, NAFLD is associated with reduced levels of dietary $\omega-3$ fatty acids, elevated plasma and hepatic SFAs; therefore, reducing the daily dietary intake of SFAs and provision of $\omega-3$ fatty acids improve NAFLD in children [78-80]. Interestingly, various studies reported that $\omega-3$ fatty acid supplementation for 6 months in NAFLD children did not improve ALT levels [81-83]; however, Nobili et al. (2014) provided $\omega-3$ fatty acids to NAFLD children for 18 months and a significant improvement in ALT levels was observed [80]. The observed effects of $\omega-3$ fatty acids after an 18-month treatment on ALT levels suggest that $\omega-3$ fatty acids are effective against ALT levels but needs prolonged treatment and therefore we don't see the effects of $\omega-3$ fatty acids in a 6-month treatment on serum ALT levels. Importantly, $\omega-3$ fatty acids improve lipid profile, insulin 
sensitivity and reduce plasma TG levels, hepatic steatosis and cytokines production [79]. Recently, it has been reported that DHA has the potential to reduce markers of NAFLD [84]. Likewise, $\omega-3$ fatty acids improve gut mirobiota that are beneficial against genes involved in lipogenesis in high carbohydrate diet-induced steatosic mice [85]. DHA act on G protein-coupled receptor 120 (GPR-120), and has shown beneficial affects against NAFLD in children [80]. Additionally, reduced hepatic proginator cells and macrophages and up-regulated GPR 120 expression after DHA supplementation [80] suggest that DHA modulate hepatic progenitor cells and macrophages through activated GPR-120 signalling. Nobili et al. (2011) reported that $\omega-3$ improves insulin sensitivity and hepatic echogenicity in children suffering from NAFLD [86]. Although it has been suggested that it is premature to describe $\omega-3$ as a treatment for NASH and NAFLD subjects, it should be the first line therapeutic agent to treat hypertriglyceridic NAFLD patients [78]. Further studies are required to confirm whether $\omega-3$ is suitable for the treatment of NASH and NAFLD in humans.

\section{Trans fatty acids}

Trans fatty acids are produced in natural foods as a result of bacterial metabolism (dairy products) and hydrogenation (margarines) and may have a role in NAFLD pathogenesis. Trans fatty acids consist of many isomers that differentially regulate the human metabolism [10]. It has been reported that Cis-9, trans- 11 conjugated linoleic acid and trans- 11 oleic acid, derived from bacterial metabolism and existing in dairy products, have no adverse effects on lipoprotein levels [87]. Paradoxically, it has been reported that the intake of trans-10, cis-12 conjugated linoleic acid present in hydrogenated oils increases inflammatory markers in women [88], causes endothelial dysfunction [89], and adversely affects the plasma lipid profile by increasing LDL:HDL and total cholesterol (TC):HDL ratios in humans [90]. Although the exact role of trans fatty acids on the lipid profile and its mechanism of action is not yet clear, dietary recommendations in NAFLD suggest avoiding highly processed food products enriched with trans fatty acids [91]. Further pre-clinical and clinical studies are required to unveil the role of trans fats in NAFLD pathogenesis.

\section{Proteins}

The role of high protein intake in causing various complications including intrarenal capillary hypertension, glomerular sclerosis, and finally renal dysfunction in some susceptible individuals [92-96] has been studied; however, studies about the role of proteins in NAFLD pathogenesis are limited. Clinical studies have reported that protein intake affects the pathogenesis of NAFLD (for a review, see [74]). Malnutrition and protein deficiency induce hepatic steatosis and NASH [97, 98]. By inhibiting de nove lipogenesis, high protein and low carbohydrates diets improve carbohydrate metabolism and liver steatosis [74]. Dietary protein intake is important for hepatocyte regeneration and provides important amino acids that prevent fat deposition in the liver [98]. Arciero et al. (2008) proved that a moderate protein diet (25\% energy from protein) has no side effects and reduces body fat content as much as a high protein diet does and, therefore, they recommended a moderate protein diet for NAFLD patients [99]. Likewise, recently it was reported that proteins that exist in dietary mung beans are beneficial against HFD-induced NAFLD in male mice [14]. Duarte et al. (2014) studied the effects of a high protein, low carbohydrate diet in adult NAFLD patients. They found no change in body weight, weight circumference and body fat. Interestingly the diet showed increased HDL-cholesterol and decreased total LDL and VLDL cholesterol, TGs, AST, gamma glutamyltransferase (GGT), alkaline phos-phatase (AP) and fasting blood glucose [12]. This study suggests that independent of its effect on body weight and body fat, a high protein, low carbohydrate diet improves the lipid profile, insulin homeostasis and liver enzymes. Furthermore, soy protein has shown positive effects against NASH by reducing plasma cholesterol levels and fat deposition in body [100]. Additionally, soy protein also reduced TGs deposition in the liver and insulin sensitivity and antioxidant activities in rats [100]. To study the role of soy protein in NAFLD subjects, Kani et al. (2014) gave a low caloric, low carbohydrate soy containing diet to adult NAFLD patients. It was found that a soy containing diet reduced ALT levels, but the lipid profile was not affected [13].

\section{Micronutrients}

Micronutrients are different from macronutrients, as the body only requires them in very small amounts. Micronutrients consist of vitamins and minerals; however here, we will discuss the role of some important micronutrients in the prevention of NAFLD development (Fig. 3).

\section{Vitamin $\mathrm{E}$}

Mitochondrial stress triggered by ROS is one of the etiological mechanisms of NAFLD pathogenesis (Fig. 1); therefore, antioxidants, for example, vitamin E, might have beneficial effects against NAFLD. Lavine was the first to propose vitamin E, a potent 
fat-soluble antioxidant, as an adjunct therapy for NAFLD. He checked the effects of vitamin E against high levels of ALT and hepatic steatosis in children [101]. Subsequently, various studies have used vitamin E for NAFLD treatment. Many groups reported that vitamin $\mathrm{E}$ treatment is ineffective to improve ALT levels in biopsy proven $[102,103]$ and suspected NAFLD [104] children. Similarly, we studied the effects of vitamin E and lifestyle on ALT levels in Chinese obese children suffering from NAFLD [105]. Paradoxically, it was found that vitamin $\mathrm{E}$ is effective in reducing ALT levels in children; however the improvement was less compared to a summer camp lifestyle program. Therefore, these studies [103, 105] collectively suggest that lifestyle is more beneficial than vitamin $\mathrm{E}$ supplementation to improve ALT levels. The positive effects (opposite to other studies) of vitamin $\mathrm{E}$ in our study are not clear but it can be speculated that the different experimental design, food and ethnicity may have some effects on vitamin-induced changes in ALT levels; however further studies are required to confirm it. Additionally, vitamin E, DHA, and choline combination reduces ALT and glucose levels and improves steatosis in NASH children. As DHA, vitamin $\mathrm{E}$ and choline all have beneficial effects against NAFLD, it is difficult to say which component has improved the conditions. Additionally, reduced hepatic steatosis, inflammation, hepatocyte ballooning, and aminotransferase levels were reported after vitamin E supplementation in NASH patients [106]. Vitamin $\mathrm{E}$ is effective against steatoheptitis in NAFLD patients but is ineffective against hepatic fibrosis once it is established [107]. Furthermore, supplementation of vitamin $\mathrm{E}$ and ascorbic acid with a modified diet and physical exercise has improved liver function and glucose metabolism in children [103]. Additionally, vitamin E supplementation with a healthy lifestyle was effective against hepatocytes ballooning; however, aminotransferase levels were not affected but a significant improvement in NAS score and resolution of NASH was observed in both adults and children [102]. Importantly, one should be very careful during prescription of vitamin $\mathrm{E}$ as high doses of vitamin $\mathrm{E}$ induce complications including some cancer types [102].

The European Association for the Study of the Liver (EASL) advises vitamin $\mathrm{E}$ as a first line therapeutic agent for non-diabetic adults with biopsy proven NASH but the American Association for the Study of Liver Diseases (AASLD) guidelines suggest that although vitamin E supplementation is effective against non-diabetic NASH in children, further confirmatory trials are required before using it in clinical practice.

\section{Vitamin C}

Being an antioxidant, vitamin $\mathrm{C}$ might be beneficial against NAFLD. In this regards, Oliveira et al. (2003) induced fatty liver disease in Wistar rats by feeding them with choline deficient diet[15]. To investigate the role of vitamin $\mathrm{C}$ (potent hydrosoluble antioxidant) against fatty liver disease, Oliveira et al. (2003) fed fatty liver rats $(n=6)$ with vitamin C (30 $\mathrm{mg} / \mathrm{Kg} /$ day) for four weeks. The control $(\mathrm{n}=6)$ rats were fed with a placebo. It was found that vitamin $C$ treatment reduced oxidative stress and inhibited steatosis in choline-deficient diet induced fatty liver rats [15]. Therefore, the aforementioned [15] positive effects of vitamin $\mathrm{C}$ against oxidative stress and steatosis might be due to its antioxidant activity. However, another study reported that vitamin C treatment reduces plasma cholesterol and TG levels and suggests that the beneficial effects of vitamin $C$ against fatty liver are because of its anti-atherogenic action [108]. Similarly, Harrison et al. (2003) performed a prospective, double-blind, randomized placebo-controlled study and included histology based NASH in men and women. They divided the subjects into two groups, either to receive vitamins $\mathrm{E}$ and C (1000 IU and1000 $\mathrm{mg}$, respectively) or a placebo daily for 6 months [109]. It was found that vitamin E and vitamin $C$ supplementations reduce hepatic fibrosis, especially in diabetic NASH patients. However, this therapy did not affect inflammation or ALT levels [109]. Although this study showed the positive effects of vitamins (E and $C$ ) in 6-month treatments on hepatic fibrosis, it remains unknown whether this therapy will be effective if applied for a longer time and that the resulted improvement in fibrosis affects the transition of NASH to cirrhosis and hepatocellular carcinoma. Therefore, further pre-clinical and clinical trials should be done to unveil the effects of long-term vitamin $C$ treatment on fibrosis, cirrhosis, hepatocellular carcinoma, inflammation and ALT levels. It is also worthwhile investigating whether vitamin $C$ treatment improves haptic complication through its antioxidant properties or any other action.

\section{Vitamin D}

Vitamin D deficiency is associated with NAFLD pathogenesis. Obese patients have been reported to be more deficient in vitamin $\mathrm{D}$ compared to normal weight and overweight subjects [106, 110-112]. Vitamin D regulates various genes widely distributed in the liver and some of them are involved in glucose and fat metabolism [106, 110]. Through the activation of TLRs, vitamin D deficiency exacerbates NAFLD and has association with hepatic inflammatory markers, oxidative stress and insulin resistance in rats 
[113]. Importantly, reduced plasma vitamin D levels have an association with insulin resistance and T2D and proper supplementation of vitamin D improves insulin sensitivity [114]. Similarly independent of fat deposition and insulin resistance, reduced levels of plasma vitamin D has an association with NAFLD in adults [110]. Interestingly, vitamin D deficiency [115] and increased prevalence of NAFLD in obese children [4] link vitamin D deficiency and NAFLD occurrence. In this regard, Misra et al. (2008) studied the association between vitamin D and NAFLD in children. Vitamin D deficiency was reported in NAFLD patients, but vitamin D deficiency was not associated with NAFLD severity [115]. Similarly, Black et al. (2014) also reported vitamin D deficiency in NAFLD adult patients but the serum vitamin D levels were inversely proportional to NAFLD severity [110]. Additionally, the same inverse proportion between serum vitamin D levels and NAFLD severity has been reported in children with high ALT levels and a hyperchogenic liver [116]. The above studies clearly showed vitamin D deficiency in NAFLD patients and opened a new window for researchers to study the role of vitamin D deficiency in NAFLD pathogenesis and then to use vitamin $\mathrm{D}$ as an adjunct therapy for NAFLD treatment.

In this regard, one study used vitamin D supplementation against adult NAFLD subjects and reported reduced high-sensitive $\mathrm{C}$-reactive protein (hs-CRP) and malondialdehyde (MDA) levels but ALT levels were not affected [117]. However, further pre-clinical and clinical studies are suggested to investigate the relation of vitamin $\mathrm{D}$ with insulin resistant, adiposity and NAFLD and whether supplementation of vitamin $\mathrm{D}$ is beneficial against NAFLD development and progression.

\section{Polyphenol}

It was reported that polyphenols have been used for NAFLD treatment (Aguirre et al., 2014). Resveratrol, a member of the polyphenols family has shown anti-steatotic, anti-inflammatory, and antioxidative effects (for a review, see [118]). In this regard, various studies have reported that resveratrol supplementations reduce liver enzymes and inflammatory cytokines $[119,120]$ and hepatic steatosis [121]. Similarly, Chen et al. (2015) randomized adult NAFLD subjects into two groups: ( 2 capsules with $150 \mathrm{mg}$ of resveratrol/day) as a treatment and (2 capsules of placebo/day) as a control. It was found that a treatment of 2 capsules with $150 \mathrm{mg}$ of resveratrol/day significantly reduced AST, ALT, LDL cholesterol and TC levels and improved the glucose profile compared to the control group [120]. Similarly, Faghihzadeh et al. (2014) also divided adult male and female NAFLD patients into two groups, control (placebo) and intervention (500 $\mathrm{mg}$ of resveratrol/day) with a balanced diet and healthy lifestyle. It was found that resveratrol treatment improves various parameters of NAFLD pathogenesis [119]. Various other members of the polyphenol family, including curcumin [122], quercetin [119], anthocyanin [122] and green tea polyphenols [123], also showed beneficial effects in the treatment of adult NAFLD subjects. The beneficial effects of polyphenols in adult NAFLD subjects hint at its beneficial effects in children but careful pre-clinical and clinical studies are required to test this hypothesis.

\section{Summary}

NAFLD is a an acquired metabolic liver disease characterized by TGs deposition in more than 5\% hepatocytes not induced by alcohol consumption and other etiologic factors causing liver diseases, including drugs, toxins, infectious diseases, etc.[124]. NAFLD ranges from simple steatosis to non-alcoholic steatohepatitis (NASH) that lead to liver fibrosis, cirrhosis, and hepatocellular carcinoma [125].

Various factors including genetic and epigenetic pathways, sedentary lifestyle, sleep, and high energy diets play a key role in NAFLD and NASH pathogenesis [33, 126, 127]. Oxidative stress, liver inflammation, mitochondrial dysfunction, imbalanced pro-inflammatory cytokines, fibrosis, insulin resistance, hyperinsulinemia, plasma FFAs, fatty liver, and hepatocyte injury are pathologies that support the development of NASH and fibrogenesis [16-20]. Nutrition plays a key role in the pathogenesis of NAFLD. Among macronutrients, fat and carbohydrate rich diets support/prevent the pathogenesis of NAFLD [7, 39, 61, 128]. Fructose from carbohydrates $[48,49]$, as well as SFAs [62, 128], trans fatty acids [88] and $\omega-6$ fatty acids [11] from fats, support NAFLD pathogenesis. Furthermore, fiber and low glycemic index diets from carbohydrates [53, 55], as well as MUFAs [72] and $\omega-3$ fatty acids from fats, prevent NAFLD pathogenesis $[80,84]$. Importantly, proteins have beneficial effects against NAFLD [99, 128]. Among micronutrients, vitamin C [109], vitamin E [102-104], vitamin D [117] and polyphenols [119, 120] prevent NAFLD pathogenesis. However, many research areas about NAFLD are still new and need further experimentations, including the role of vitamin $C$, vitamin $D, \omega-3 / \omega-6$ fatty acids and proteins in the pathogenesis/prevention of NAFLD. Similarly, further pre-clinical and clinical trials will be helpful to unveil the exact role and mode of action of high fiber and low glycemic diets in NAFLD pathogenesis. 


\section{Abbreviations}

NAFLD: non-alcoholic fatty liver disease; TGs: triglycerides; NAFL: non-alcoholic fatty liver; FFAs: free fatty acids; TLRs: toll like receptors; NASH: non-alcoholic steatohepatitis; HPCs: hepatic progenitor cells; IL: interleukin, PPAR-a: perioxisome proliferator-activated receptor- $\alpha$; ROS: reactive oxygen species; ALT: alanine aminotransferase; TNF-a: tumour necrosis factor alpha; AST: aspartate aminotransferase; SFAs: saturated fatty acids; ER: endoplasmic reticulum; LDL: low density lipoprotein; HDL: high density lipoprotein; TC: total cholesterol; PUFAs: polyunsaturated fatty acids; GGT: gamma glutamyltransferase, AP: alkaline phos-phatase; BMI: body mass index; GI: low-glycemic index; MUFAs: monounsaturated fatty acids, VLD: very low density lipoprotein; SREBP: sterol regulatory element binding protein; $\omega$-3: omega 3; DHA: docosahexaenoic acid; EPA: eicosapentaenoic acid; GPR-120: G proteincoupled receptor 120; EASL; European Association for the Study of the Liver; AASLD: American Association for the Study of Liver Diseases; MDA: malondialdehyde; hs-CRP: high-sensitive C-reactive protein.

\section{Acknowledgements}

The authors gratefully acknowledge the financial support from the National Key Research and Development Programme of China (No. 2016YFC1305 301), National Natural Science Foundation of China (Nos. 81570759 and 81270938), Zhejiang Provincial Key Science and Technology Project (No. 2014C030452), Key Disciplines of Medicine (Innovation discipline, 11-CX24) and the Fundamental Research Funds for the Central Universities (2017XZZX001-01). No specific grant from any funding agency, commercial or not-for-profit sectors supported the current work.

\section{Competing Interests}

The authors have declared that no competing interest exists.

\section{References}

1. Fan JG, Jia JD, Li YM, Wang BY, Lu LG, Shi JP, et al. Guidelines for the diagnosis and management of nonalcoholic fatty liver disease: update 2010. Journal of digestive diseases. 2011; 12: 38-44.

2. Clark JM. The epidemiology of nonalcoholic fatty liver disease in adults. Journal of Clinical Gastroenterology. 2006; 40: S5-S10.

3. Lazo M, Clark JM. The Epidemiology of Nonalcoholic Fatty Liver Disease: A Global Perspective. Seminars in Liver Disease. 2008; 28: 339-50.

4. Bellentani S, Scaglioni F, Marino M, Bedogni G. Epidemiology of Non-Alcoholic Fatty Liver Disease. Digestive Diseases. 2010; 28: 155-61.

5. Rahimlou M, Ahmadnia H, Hekmatdoost A. Dietary supplements and pediatric non-alcoholic fatty liver disease: Present and the future. World Journal of Hepatology. 2015; 7: 2597-602.

6. Spruss A, Bergheim I. Dietary fructose and intestinal barrier: potential risk factor in the pathogenesis of nonalcoholic fatty liver disease. Journal of Nutritional Biochemistry. 2009; 20: 657-62.
7. Semiane N, Foufelle F, Ferré P, Hainault I, Ameddah S, Mallek A, et al. High carbohydrate diet induces nonalcoholic steato-hepatitis (NASH) in a desert gerbil. Comptes Rendus Biologies. 2017; 340: 25-36.

8. Hu Y, Block G, Norkus EP, Morrow JD, Dietrich M, Hudes M. Relations of glycemic index and glycemic load with plasma oxidative stress markers. American Journal of Clinical Nutrition. 2006; 84: 70-6.

9. Dongiovanni P, Lanti C, Gatti S, Rametta R, Recalcati S, Maggioni M, et al. High fat diet subverts hepatocellular iron uptake determining dysmetabolic iron overload. PloS one. 2015; 10: e0116855.

10. Mensink RP. Metabolic and health effects of isomeric fatty acids. Current Opinion in Lipidology. 2005; 16: 27-30.

11. Reiner Ž, Catapano AL, De Backer G, Graham I, Taskinen M-R, Wiklund $\mathrm{O}$, et al. ESC/EAS Guidelines for the management of dyslipidaemias. European heart journal. 2011; 32: 1769-818.

12. Duarte SMB, Faintuch J, Stefano JT, OLIVEIRA MBSd, MAZO DFdC, Rabelo F, et al. Hypocaloric high-protein diet improves clinical and biochemical markers in patients with nonalcoholic fatty liver disease (NAFLD). Nutricion hospitalaria. 2014; 29: 94-101.

13. Kani AH, Alavian SM, Esmaillzadeh A, Adibi P, Azadbakht L. Effects of a novel therapeutic diet on liver enzymes and coagulating factors in patients with non-alcoholic fatty liver disease: A parallel randomized trial. Nutrition. 2014; 30: 814-21.

14. Watanabe H, Inaba $\mathrm{Y}$, Kimura K, Asahara S-i, Kido Y, Matsumoto M, et al. Dietary Mung Bean Protein Reduces Hepatic Steatosis, Fibrosis, and Inflammation in Male Mice with Diet-Induced, Nonalcoholic Fatty Liver Disease. The Journal of Nutrition. 2017; 147: 52-60.

15. Oliveira CP, da Costa Gayotto LC, Tatai C, Della Nina BI, Lima ES, Abdalla DS, et al. Vitamin C and vitamin $\mathrm{E}$ in prevention of nonalcoholic fatty liver disease (NAFLD) in choline deficient diet fed rats. Nutrition journal. 2003; 2 : 9 .

16. Berardis S, Sokal E. Pediatric non-alcoholic fatty liver disease: an increasing public health issue. European Journal of Pediatrics. 2014; 173: 131-9.

17. Conlon B, Beasley J, Aebersold K, Jhangiani S, Wylie-Rosett J. Nutritional Management of Insulin Resistance in Nonalcoholic Fatty Liver Disease (NAFLD). Nutrients. 2013; 5: 4093.

18. Yang M, Gong S, Ye S, Lyman B, Geng L, Chen P, et al. Non-Alcoholic Fatty Liver Disease in Children: Focus on Nutritional Interventions. Nutrients. 2014; 6: 4691.

19. Dowman JK, Tomlinson JW, Newsome PN. Pathogenesis of non-alcoholic fatty liver disease. QJM: An International Journal of Medicine. 2010; 103: 71-83.

20. Takaki A, Kawai D, Yamamoto K. Multiple Hits, Including Oxidative Stress, as Pathogenesis and Treatment Target in Non-Alcoholic Steatohepatitis (NASH). International Journal of Molecular Sciences. 2013; 14: 20704-28.

21. Ayonrinde OT, Olynyk JK, Marsh JA, Beilin LJ, Mori TA, Oddy WH, et al. Childhood adiposity trajectories and risk of nonalcoholic fatty liver disease in adolescents. Journal of Gastroenterology and Hepatology. 2015; 30: 163-71.

22. Holterman A-XL, Guzman G, Fantuzzi G, Wang H, Aigner K, Browne A, et al. Nonalcoholic fatty liver disease in severely obese adolescent and adult patients. Obesity. 2013; 21: 591-7.

23. Ferreyra Solari NE, Inzaugarat ME, Baz P, De Matteo E, Lezama C, Galoppo M, et al. The Role of Innate Cells Is Coupled to a Th1-Polarized Immune Response in Pediatric Nonalcoholic Steatohepatitis. Journal of Clinical Immunology. 2012; 32: 611-21.

24. De Vito R, Alisi A, Masotti A, Ceccarelli S, Panera N, Citti A, et al. Markers of activated inflammatory cells correlate with severity of liver damage in children with nonalcoholic fatty liver disease. International Journal of Molecular Medicine. 2012; 30: 49-56.

25. Chen WEI, Wang X, Huang LI, Liu BO. Hepcidin in non-alcoholic fatty liver disease regulated by the TLR4/NF-KB signaling pathway. Experimental and Therapeutic Medicine. 2016; 11: 73-6.

26. Dattaroy D, Seth RK, Das S, Alhasson F, Chandrashekaran V, Michelotti $\mathrm{G}$, et al. Sparstolonin B attenuates early liver inflammation in experimental NASH by modulating TLR4 trafficking in lipid rafts via NADPH oxidase activation. American Journal of Physiology Gastrointestinal and Liver Physiology. 2016; 310: G510-G25.

27. Nobili V, Carpino G, Alisi A, Franchitto A, Alpini G, De Vito R, et al. Hepatic progenitor cells activation, fibrosis, and adipokines production in pediatric nonalcoholic fatty liver disease. Hepatology. 2012; 56: 2142-53.

28. Mouralidarane A, Soeda J, Visconti-Pugmire C, Samuelsson A-M, Pombo J, Maragkoudaki X, et al. Maternal Obesity Programs Offspring Nonalcoholic Fatty Liver Disease by Innate Immune Dysfunction in Mice. Hepatology. 2013; 58: 128-38.

29. Stienstra R, Saudale F, Duval C, Keshtkar S, Groener JEM, van Rooijen N, et al. Kupffer Cells Promote Hepatic Steatosis Via Interleukin-1 
beta-Dependent Suppression of Peroxisome Proliferator-Activated Receptor alpha Activity. Hepatology. 2010; 51: 511-22.

30. Chalasani N, Younossi Z, Lavine JE, Diehl AM, Brunt EM, Cusi K, et al. The Diagnosis and Management of Non-alcoholic Fatty Liver Disease: Practice Guideline by the American Association for the Study of Liver Diseases, American College of Gastroenterology, and the American Gastroenterological Association. American Journal of Gastroenterology. 2012; 107: 811-26.

31. Morgan ML, Sigala B, Soeda J, Cordero P, Nguyen V, McKee C, et al. Acetylcholine induces fibrogenic effects via M2/M3 acetylcholine receptors in non-alcoholic steatohepatitis and in primary human hepatic stellate cells. Journal of Gastroenterology and Hepatology. 2016; 31: 475-83.

32. Sigala B, McKee C, Soeda J, Pazienza V, Morgan M, Lin C-I, et al. Sympathetic Nervous System Catecholamines and Neuropeptide $Y$ Neurotransmitters Are Upregulated in Human NAFLD and Modulate the Fibrogenic Function of Hepatic Stellate Cells. Plos One. 2013; 8.

33. Nobili V, Svegliati-Baroni G, Alisi A, Miele L, Valenti L, Vajro P. A 360-degree overview of paediatric NAFLD: Recent insights. Journal of Hepatology. 2013; 58: 1218-29.

34. Alisi A, Carpino G, Nobili V. Paediatric nonalcoholic fatty liver disease. Current opinion in gastroenterology. 2013; 29: 279-84.

35. Schwimmer JB, Behling C, Newbury R, Deutsch R, Nievergelt C, Schork $\mathrm{NJ}$, et al. Histopathology of pediatric nonalcoholic fatty liver disease. Hepatology. 2005; 42: 641-9.

36. Betancourt-Garcia MM, Arguelles A, Montes J, Hernandez A, Singh M, Forse RA. Pediatric Nonalcoholic Fatty Liver Disease: the Rise of a Lethal Disease Among Mexican American Hispanic Children. Obesity Surgery. 2017; 27: 236-44.

37. Loomba R, Sirlin CB, Schwimmer JB, Lavine JE. Advances in Pediatric Nonalcoholic Fatty Liver Disease. Hepatology (Baltimore, Md). 2009; 50: 1282-93.

38. Nobili V, Alisi A, Newton KP, Schwimmer JB. Comparison of the Phenotype and Approach to Pediatric Versus Adult Patients with Nonalcoholic Fatty Liver Disease. Gastroenterology. 2016; 150: 1798-810.

39. Neuschwander T, Brent A. Carbohydrate intake and nonalcoholic fatty liver disease. Current Opinion in Clinical Nutrition \& Metabolic Care. 2013; 16: 446-52.

40. Schugar RC, Crawford PA. Low-carbohydrate ketogenic diets, glucose homeostasis, and nonalcoholic fatty liver disease. Current opinion in clinical nutrition and metabolic care. 2012;15: 374.

41. Browning JD, Baker JA, Rogers T, Davis J, Satapati S, Burgess SC. Short-term weight loss and hepatic triglyceride reduction: evidence of a metabolic advantage with dietary carbohydrate restriction. American Journal of Clinical Nutrition. 2011; 93: 1048-52.

42. Foster GD, Wyatt HR, Hill JO, Makris AP, Rosenbaum DL, Brill C, et al. Weight and Metabolic Outcomes After 2 Years on a Low-Carbohydrate Versus Low-Fat Diet A Randomized Trial. Annals of Internal Medicine. 2010; 153: 147-U37.

43. Hu T, Mills KT, Yao L, Demanelis K, Eloustaz M, Yancy WS, et al. Effects of low-carbohydrate diets versus low-fat diets on metabolic risk factors: a meta-analysis of randomized controlled clinical trials. American journal of epidemiology. 2012; 176: S44-S54.

44. Jin R, Ngoc-Anh L, Liu S, Epperson MF, Ziegler TR, Welsh JA, et al. Children with NAFLD Are More Sensitive to the Adverse Metabolic Effects of Fructose Beverages than Children without NAFLD. Journal of Clinical Endocrinology \& Metabolism. 2012; 97: E1088-E98.

45. Nseir W, Nassar F, Assy N. Soft drinks consumption and nonalcoholic fatty liver disease. World Journal of Gastroenterology : WJG. 2010; 16: 2579-88.

46. Abid A, Taha O, Nseir W, Farah R, Grosovski M, Assy N. Soft drink consumption is associated with fatty liver disease independent of metabolic syndrome. Journal of Hepatology. 2009; 51: 918-24.

47. Zivkovic AM, German JB, Sanyal AJ. Comparative review of diets for the metabolic syndrome: implications for nonalcoholic fatty liver disease. The American journal of clinical nutrition. 2007; 86: 285-300.

48. Roglans N, Vila L, Farre M, Alegret M, Maria Sanchez R, Vaquez-Carrera $\mathrm{M}$, et al. Impairment of hepatic STAT-3 activation and reduction of PPAR alpha activity in fructose-fed rats. Hepatology. 2007; 45: 778-88.

49. Le K-A, Bortolotti M. Role of dietary carbohydrates and macronutrients in the pathogenesis of nonalcoholic fatty liver disease. Current Opinion in Clinical Nutrition and Metabolic Care. 2008; 11: 477-82.

50. Dhingra R, Sullivan L, Jacques PF, Wang TJ, Fox CS, Meigs JB, et al. Soft Drink Consumption and Risk of Developing Cardiometabolic Risk Factors and the Metabolic Syndrome in Middle-Aged Adults in the Community. Circulation. 2007; 116: 480

51. Jenkins DJ, Kendall CW, Vuksan V, Vidgen E, Parker T, Faulkner D, et al. Soluble fiber intake at a dose approved by the US Food and Drug Administration for a claim of health benefits: serum lipid risk factors for cardiovascular disease assessed in a randomized controlled crossover trial. The American journal of clinical nutrition. 2002; 75: 834-9.

52. Naumann E, VaN Rees AB, Önning G, Öste R, Wydra M, Mensink RP. $\beta$-Glucan incorporated into a fruit drink effectively lowers serum LDL-cholesterol concentrations. The American journal of clinical nutrition. 2006; 83: 601-5.

53. Anderson JW, Randles KM, Kendall CW, Jenkins DJ. Carbohydrate and fiber recommendations for individuals with diabetes: a quantitative assessment and meta-analysis of the evidence. Journal of the American College of Nutrition. 2004; 23: 5-17.

54. Jenkins D, Wolever T, Taylor RH, Griffiths C, Krzeminska K, Lawrie JA, et al. Slow release dietary carbohydrate improves second meal tolerance. The American journal of clinical nutrition. 1982; 35: 1339-46.

55. Brighenti F, Benini L, Del Rio D, Casiraghi C, Pellegrini N, Scazzina F, et al. Colonic fermentation of indigestible carbohydrates contributes to the second-meal effect. The American journal of clinical nutrition. 2006; 83: $817-22$

56. Xu AM, Wang Y, Keshaw H, Xu LY, Lam KSL, Cooper GIS. The fat-derived hormone adiponectin alleviates alcoholic and nonalcoholic fatty liver diseases in mice. Journal of Clinical Investigation. 2003; 112: 91-100.

57. Fu J-F, Fang Y-L, Liang L, Wang C-L, Hong F, Dong G-P. A rabbit model of pediatric nonalcoholic steatohepatitis: the role of adiponectin. World J Gastroenterol. 2009; 15: 912-8.

58. Fang Y, Liang L, Fu J. Establishment of rabbit model of juvenile nonalcoholic steatohepatitis. Zhejiang da xue xue bao Yi xue ban= Journal of Zhejiang University Medical sciences. 2008; 37: 240-4.

59. Fang $\mathrm{Y}$, Liang $\mathrm{L}, \mathrm{Fu}$ J. The relationship between adiponectin and nonalcoholic fatty liver disease in infant rabbits fed a fat-rich diet. Zhonghua gan zang bing za zhi= Zhonghua ganzangbing zazhi= Chinese journal of hepatology. 2007; 15: 316-7.

60. Liang L, Fu J, Zou C, Hong F, Wang C, Wang X. Metformin hydrochloride ameliorates adiponectin levels and insulin sensitivity in adolescents with metabolic syndrome. Zhonghua er ke za zhi Chinese journal of pediatrics. 2006; 44: 118-21

61. Shi $\mathrm{H}, \mathrm{Fu} \mathrm{J}, \mathrm{Wang} \mathrm{C}$. Clinical value of hepatic fibrosis parameters and serum ferritin in obese children with nonalcoholic fatty liver disease. Zhejiang da xue xue bao Yi xue ban= Journal of Zhejiang University Medical sciences. 2008; 37: 245-9.

62. Wang D, Wei YR, Pagliassotti MJ. Saturated fatty acids promote endoplasmic reticulum stress and liver injury in rats with hepatic steatosis. Endocrinology. 2006; 147: 943-51.

63. Milanski M, Degasperi G, Coope A, Morari J, Denis R, Cintra DE, et al. Saturated fatty acids produce an inflammatory response predominantly through the activation of TLR4 signaling in hypothalamus: implications for the pathogenesis of obesity. J Neurosci. 2009; 29: 359-70.

64. Kleinridders A, Schenten D, Könner AC, Belgardt BF, Mauer J, Okamura T, et al. MyD88 Signaling in the CNS Is Required for Development of Fatty Acid-Induced Leptin Resistance and Diet-Induced Obesity. Cell Metabolism. 2009; 10: 249-59.

65. Ste Marie L, Miura GI, Marsh DJ, Yagaloff K, Palmiter RD. A metabolic defect promotes obesity in mice lacking melanocortin-4 receptors. Proceedings of the National Academy of Sciences of the United States of America. 2000; 97: 12339-44.

66. Lapointe A, Couillard C, Lemieux S. Effects of dietary factors on oxidation of low-density lipoprotein particles. The Journal of Nutritional Biochemistry. 2006; 17: 645-58.

67. Sacks FM, Katan M. Randomized clinical trials on the effects of dietary fat and carbohydrate on plasma lipoproteins and cardiovascular disease. The American journal of medicine. 2002; 113: 13-24.

68. Williams C. Beneficial nutritional properties of olive oil: implications for postprandial lipoproteins and factor VII. Nutrition, metabolism, and cardiovascular diseases: NMCD. 2001; 11: 51-6.

69. Rajaram S, Burke K, Connell B, Myint T, Sabaté J. A monounsaturated fatty acid-rich pecan-enriched diet favorably alters the serum lipid profile of healthy men and women. The Journal of nutrition. 2001; 131: 2275-9.

70. Kris-Etherton PM, Pearson TA, Wan Y, Hargrove RL, Moriarty K, Fishell $\mathrm{V}$, et al. High-monounsaturated fatty acid diets lower both plasma cholesterol and triacylglycerol concentrations. The American journal of clinical nutrition. 1999; 70: 1009-15.

71. Julius U. Fat modification in the diabetes diet. Experimental and clinical endocrinology \& diabetes. 2003; 111: 60-5.

72. Bozzetto L, Prinster A, Annuzzi G, Costagliola L, Mangione A, Vitelli A, et al. Liver fat is reduced by an isoenergetic MUFA diet in a controlled randomized study in type 2 diabetic patients. Diabetes care. 2012; 35: 1429-35.

73. Kruse M, von Loeffelholz C, Hoffmann D, Pohlmann A, Seltmann AC, Osterhoff $\mathrm{M}$, et al. Dietary rapeseed/canola-oil supplementation reduces 
serum lipids and liver enzymes and alters postprandial inflammatory responses in adipose tissue compared to olive-oil supplementation in obese men. Molecular nutrition \& food research. 2015; 59: 507-19.

74. Kargulewicz A, Stankowiak-Kulpa H, Grzymisławski M. Dietary recommendations for patients with nonalcoholic fatty liver disease. Przeglad Gastroenterologiczny. 2014; 9: 18-23.

75. Alisi A, Nobili V. Non-alcoholic fatty liver disease in children now: Lifestyle changes and pharmacologic treatments. Nutrition. 2012; 28: 722-6.

76. Flachs P, Rossmeisl M, Bryhn M, Kopecky J. Cellular and molecular effects of n-3 polyunsaturated fatty acids on adipose tissue biology and metabolism. Clinical Science. 2009; 116: 1-16.

77. Masterton GS, Plevris JN, Hayes PC. Review article: omega-3 fatty acids a promising novel therapy for non-alcoholic fatty liver disease. Alimentary Pharmacology \& Therapeutics. 2010; 31: 679-92.

78. Mencin AA, Lavine JE. Advances in Pediatric Nonalcoholic Fatty Liver Disease. Pediatric Clinics of North America. 2011; 58: 1375-92.

79. Janczyk W, Socha P, Lebensztejn D, Wierzbicka A, Mazur A, Neuhoff-Murawska J, et al. Omega-3 fatty acids for treatment of non-alcoholic fatty liver disease: design and rationale of randomized controlled trial. Bmc Pediatrics. 2013; 13.

80. Nobili V, Carpino G, Alisi A, De Vito R, Franchitto A, Alpini G, et al. Role of Docosahexaenoic Acid Treatment in Improving Liver Histology in Pediatric Nonalcoholic Fatty Liver Disease. Plos One. 2014; 9.

81. Nobili V, Bedogni G, Alisi A, Pietrobattista A, Rise P, Galli C, et al. Docosahexaenoic acid supplementation decreases liver fat content in children with non-alcoholic fatty liver disease: double-blind randomised controlled clinical trial. Archives of Disease in Childhood. 2011; 96: 350-3.

82. Pacifico L, Bonci E, Di Martino M, Versacci P, Andreoli G, Silvestri LM, et al. A double-blind, placebo-controlled randomized trial to evaluate the efficacy of docosahexaenoic acid supplementation on hepatic fat and associated cardiovascular risk factors in overweight children with nonalcoholic fatty liver disease. Nutrition, Metabolism and Cardiovascular Diseases. 2015; 25: 734-41.

83. Janczyk W, Lebensztejn D, Wierzbicka-Rucińska A, Mazur A, Neuhoff-Murawska J, Matusik P, et al. Omega-3 Fatty Acids Therapy in Children with Nonalcoholic Fatty Liver Disease: A Randomized Controlled Trial. The Journal of Pediatrics. 2015; 166: 1358-63.e3.

84. Baum JI, Carroll TG, Shouse SA. Docosahexaenoic Acid (DHA), not Leucine, May Protect HepG2 Cells from Palmitate-Induced Non-Alcoholic Fatty Liver Disease. The FASEB Journal. 2017; 31: 1036.7.

85. Huang K-H, Nichols RG, Sebastian A, Albert I, Patterson AD, Ross AC. Gut Microbiota Increased by Omega-3 Fatty Acids is Negatively Correlated with Hepatic Lipid Metabolism-Associated Genes in mice with High Carbohydrate Diet-Induced Steatosis. The FASEB Journal. 2017; 31: 654.3-.3.

86. Loy JJ, Youn HA, Schwack B, Kurian M, Fielding CR, Fielding GA. Improvement in nonalcoholic fatty liver disease and metabolic syndrome in adolescents undergoing bariatric surgery. Surgery for Obesity and Related Diseases. 2015; 11: 442-9.

87. Tricon S, Burdge GC, Jones EL, Russell JJ, El-Khazen S, Moretti E, et al. Effects of dairy products naturally enriched with cis-9,trans-11 conjugated linoleic acid on the blood lipid profile in healthy middle-aged men. American Journal of Clinical Nutrition. 2006; 83: 744-53.

88. Lopez-Garcia E, Schulze MB, Meigs JB, Manson JAE, Rifai N, Stampfer MJ, et al. Consumption of Trans fatty acids is related to plasma biomarkers of inflammation and endothelial dysfunction. Journal of Nutrition. 2005; 135: 562-6.

89. Mozaffarian D. Trans fatty acids - Effects on systemic inflammation and endothelial function. Atherosclerosis Supplements. 2006; 7: 29-32.

90. Tricon S, Burdge GC, Kew S, Banerjee T, Russell JJ, Jones EL, et al. Opposing effects of cis-9,trans-11 and trans-10,cis-12 conjugated linoleic acid on blood lipids in healthy humans. American Journal of Clinical Nutrition. 2004; 80: 614-20.

91. Zivkovic AM, German JB, Sanyal AJ. Comparative review of diets for the metabolic syndrome: implications for nonalcoholic fatty liver disease. American Journal of Clinical Nutrition. 2007; 86: 285-300.

92. Ahmed F. Effect of diet on progression of chronic renal disease. Journal of the American Dietetic Association. 1991; 91: 1266-70.

93. Meyer TW, Anderson S, Brenner BM. Dietary protein intake and progressive glomerular sclerosis: the role of capillary hypertension and hyperperfusion in the progression of renal disease. Annals of internal medicine. 1983; 98: 832-8.

94. Blachley JD. The role of dietary protein in the progression and symptomatology of chronic renal failure. The American journal of the medical sciences. 1984; 288: 228-34.

95. Levey AS, Greene T, Beck GJ, Caggiula AW, Kusek JW, Hunsicker LG, et al. Dietary Protein Restriction and the Progression of Chronic Renal
Disease What Have All of the Results of the MDRD Study Shown? Journal of the American Society of Nephrology. 1999; 10: 2426-39.

96. Klahr S, Levey AS, Beck GJ, Caggiula AW, Hunsicker L, Kusek JW, et al. The effects of dietary protein restriction and blood-pressure control on the progression of chronic renal disease. New England Journal of Medicine. 1994; 330: 877-84.

97. Meghellibouchenak M, Belleville J, Boquillon M. HEPATIC STEATOSIS AND SERUM VERY LOW-DENSITY LIPOPROTEINS DURING 2 TYPES OF PROTEIN-MALNUTRITION FOLLOWED BY BALANCED REFEEDING. Nutrition. 1989; 5: 321-9.

98. Leclercq IA, Horsmans Y. Nonalcoholic fatty liver disease: the potential role of nutritional management. Current Opinion in Clinical Nutrition and Metabolic Care. 2008; 11: 766-73.

99. Arciero PJ, Gentile CL, Pressman R, Everett M, Ormsbee MJ, Martin J, et al. Moderate protein intake improves total and regional body composition and insulin sensitivity in overweight adults. Metabolism-Clinical and Experimental. 2008; 57: 757-65.

100. Yang H-Y, Tzeng Y-H, Chai C-Y, Hsieh A-T, Chen J-R, Chang L-S, et al. Soy protein retards the progression of non-alcoholic steatohepatitis via improvement of insulin resistance and steatosis. Nutrition. 2011; 27: 943-8.

101. Lavine JE. Vitamin E treatment of nonalcoholic steatohepatitis in children: A pilot study. Journal of Pediatrics. 2000; 136: 734-8.

102. Lavine JE, Schwimmer JB, Van Natta ML, Molleston JP, Murray KF, Rosenthal P, et al. Effect of Vitamin E or Metformin for Treatment of Nonalcoholic Fatty Liver Disease in Children and Adolescents The TONIC Randomized Controlled Trial. Jama-Journal of the American Medical Association. 2011; 305: 1659-68.

103. Nobili V, Manco M, Devito R, Ciampalini P, Piemonte F, Marcellini M. Effect of vitamin $\mathrm{E}$ on aminotransferase levels and insulin resistance in children with non-alcoholic fatty liver disease. Alimentary Pharmacology \& Therapeutics. 2006; 24: 1553-61.

104. Vajro P, Mandato C, Franzese A, Ciccimarra E, Lucariello S, Savoia M, et al. Vitamin E treatment in pediatric obesity-related liver disease: A randomized study. Journal of Pediatric Gastroenterology and Nutrition. 2004; 38: 48-55.

105. Wang C-L, Liang L, Fu J-F, Zou C-C, Hong F, Xue J-Z, et al. Effect of lifestyle intervention on non-alcoholic fatty liver disease in Chinese obese children. World Journal of Gastroenterology. 2008; 14: 1598-602.

106. Li J, Cordero P, Vi N, Oben JA. The Role of Vitamins in the Pathogenesis of Non-alcoholic Fatty Liver Disease. Integrative Medicine Insights. 2016; 11: 19-25.

107. Ji H-F, Sun Y, Shen L. Effect of vitamin E supplementation on aminotransferase levels in patients with NAFLD, NASH, and CHC: Results from a meta-analysis. Nutrition. 2014; 30: 986-91.

108. Okamoto K. Vitamin C intake and apolipoproteins in a healthy elderly Japanese population. Preventive medicine. 2002; 34: 364-9.

109. Harrison SA, Torgerson S, Hayashi P, Ward J, Schenker S. Vitamin E and vitamin $C$ treatment improves fibrosis in patients with nonalcoholic steatohepatitis. The American journal of gastroenterology. 2003; 98: 2485-90.

110. Black LJ, Jacoby P, She Ping-Delfos WC, Mori TA, Beilin LJ, Olynyk JK, et al. Low serum 25-hydroxyvitamin D concentrations associate with non-alcoholic fatty liver disease in adolescents independent of adiposity. Journal of Gastroenterology and Hepatology. 2014; 29: 1215-22.

111. Goktas Z, Atilgan ZTD. Serum vitamin D levels in individuals with polycystic ovary syndrome (PCOS), with or without non-alcoholic fatty liver disease (NAFLD). The FASEB Journal. 2017; 31: 968.6.

112. Kabir M, Ebrahimi-Mameghani M, Khoshbaten M, Jamali H, Abedi R. Relationship between vitamin D, PTH and insulin resistance in the development of non-alcoholic fatty liver disease (NAFLD). Malaysian Journal of Nutrition. 2016; 22: 325-34.

113. Roth CL, Elfers CT, Figlewicz DP, Melhorn SJ, Morton GJ, Hoofnagle A, et al. Vitamin D deficiency in obese rats exacerbates nonalcoholic fatty liver disease and increases hepatic resistin and toll-like receptor activation. Hepatology. 2012; 55: 1103-11.

114. George PS, Pearson ER, Witham MD. Effect of vitamin D supplementation on glycaemic control and insulin resistance: a systematic review and meta-analysis. Diabetic Medicine. 2012; 29: E142-E50.

115. Misra M, Pacaud D, Petryk A, Collett-Solberg PF, Kappy M, Lawson Wilkins Pediat Endocrine S. Vitamin D deficiency in children and its management: Review of current knowledge and recommendations. Pediatrics. 2008; 122: 398-417.

116. Nobili V, Giorgio V, Liccardo D, Bedogni G, Morino G, Alisi A, et al. Vitamin D levels and liver histological alterations in children with nonalcoholic fatty liver disease. European Journal of Endocrinology. 2014; $170: 547-53$ 
117. Sharifi N, Amani R, Hajiani E, Cheraghian B. Does vitamin D improve liver enzymes, oxidative stress, and inflammatory biomarkers in adults with non-alcoholic fatty liver disease? A randomized clinical trial. Endocrine. 2014; 47: 70-80.

118. Charytoniuk T, Drygalski K, Konstantynowicz-Nowicka K, Berk K, Chabowski A. Alternative treatment methods attenuate the development of NAFLD: A review of resveratrol molecular mechanisms and clinical trials. Nutrition. 2017; 34: 108-17.

119. Faghihzadeh F, Adibi P, Rafiei R, Hekmatdoost A. Resveratrol supplementation improves inflammatory biomarkers in patients with nonalcoholic fatty liver disease. Nutrition Research. 2014; 34: 837-43.

120. Chen S, Zhao X, Ran L, Wan J, Wang X, Qin Y, et al. Resveratrol improves insulin resistance, glucose and lipid metabolism in patients with non-alcoholic fatty liver disease: A randomized controlled trial. Digestive and Liver Disease. 2015; 47: 226-32.

121. Oliveira Andrade JM, Paraiso AF, Macedo de Oliveira MV, Eleuterio Martins AM, Neto JF, Sena Guimaraes AL, et al. Resveratrol attenuates hepatic steatosis in high-fat fed mice by decreasing lipogenesis and inflammation. Nutrition. 2014; 30: 915-9.

122. Aguirre L, Puy Portillo M, Hijona E, Bujanda L. Effects of resveratrol and other polyphenols in hepatic steatosis. World Journal of Gastroenterology. 2014; 20: 7366-80.

123. Sakata R, Nakamura T, Torimura T, Ueno T, Sata M. Green tea with high-density catechins improves liver function and fat infiltration in non-alcoholic fatty liver disease (NAFLD) patients: A double-blind placebo-controlled study. International Journal of Molecular Medicine. 2013; 32: 989-94.

124. Oldfield IV DR, Johnson D. Non-alcoholic fatty liver disease and the gut microbiota: exploring the connection. Gastro Open J. 2015; 1: 30-43.

125. Rinella ME. Nonalcoholic fatty liver disease: A systematic review. JAMA. 2015; 313: 2263-73.

126. Africa JA, Newton KP, Schwimmer JB. Lifestyle interventions including nutrition, exercise, and supplements for nonalcoholic fatty liver disease in children. Digestive diseases and sciences. 2016; 61: 1375-86.

127. Katsagoni CN, Georgoulis M, Papatheodoridis GV, Fragopoulou E, Ioannidou P, Papageorgiou M, et al. Associations Between Lifestyle Characteristics and the Presence of Nonalcoholic Fatty Liver Disease: A Case-Control Study. Metabolic syndrome and related disorders. 2017; 15: 72-9.

128. Kargulewicz A, Stankowiak-Kulpa H, Grzymisławski M. Dietary recommendations for patients with nonalcoholic fatty liver disease. Prz Gastroenterol. 2014; 9: 18-23. 